ORIGINAL ARTICLE

\title{
Indicators of haemothorax in patients with spontaneous pneumothorax
}

\author{
C-C Hsu, Y-L Wu, H-J Lin, M-P Lin, H-R Guo
}

Emerg Med J 2005;22:415-417. doi: 10.1136/emj.2003.013441

See end of article for authors' affiliations

....................

Correspondence to: $\mathrm{H}-\mathrm{J}$ Lin, Department of Emergency Medicine, ChiMei Foundation Medical Center, 901 Jung-Hua Road, Yung-Kang City, Tainan 710, Taiwan; $790001 @$ mail.chimei. org.tw

Accepted for publication 10 May 2004

\begin{abstract}
Objectives: To identify indicators and possible risk factors of haemothorax in patients with spontaneous pneumothorax.

Methods: All patients presenting to the emergency department of Chi-Mei Foundation Medical Center, Tainan, Taiwan with primary spontaneous pneumothorax between 1 January 1997 and 31 December 2002 were screened for inclusion in the present study. Of the 211 patients who qualified, eight had spontaneous haemopneumothorax (SHP) (3.79\%). The clinical data and demographic characteristics of these patients were similar to those of patients with spontaneous pneumothorax without haemothorax (SP). Results: All eight SHP patients were thin and young men (mean age 24 years and mean weight $56.1 \mathrm{~kg}$ ). Seven were smokers. The patients with SHP were taller that the patients with SP $1177.4 \mathrm{~cm} v 170.3 \mathrm{~cm}$, respectively; $p<0.01)$, and tended to have a lower body mass index (BMI) $\left(17.9 \mathrm{~kg} / \mathrm{m}^{2} v 19.6 \mathrm{~kg} / \mathrm{m}^{2}\right.$, respectively; $p=0.06)$ and higher heart rate (101.0 $v 88.0$ beats $/ \mathrm{min}$, respectively; $p=0.09)$. Clinically, patients with SHP were more likely to have dyspnoea compared with SP patients $162.5 \%$ v $26.6 \%$, respectively; $p=0.04)$ and lower levels of haemoglobin (12.8 $v 14.7 \mathrm{~g} / \mathrm{dl}$, respectively; $p=0.01)$ and haematocrit $(38.1 \%$ v $44.1 \%$, respectively; $\mathrm{p}<0.01)$. Chest $x$ rays revealed pleural effusion in all patients with SHP but in none with SP.

Conclusions: Patients with SHP are taller, with lower levels of haemoglobin and haematocrit, and are more likely to have dyspnoea than patients without haemothorax. The chest $x$ ray finding of pneumothorax with an ipsilateral air-fluid level is a strong indicator of SHP.
\end{abstract}

S pontaneous haemopneumothorax (SHP) is a rare potentially life-threatening condition that occurs predominantly in adolescent and adult males, and constitutes $1-12 \%$ of all spontaneous pneumothoraces. ${ }^{12}$ Its major clinical features are chest pain, dyspnoea, anaemia, and, sometimes, shock, which can be dramatic and depends on the amount of haemorrhage. In SHP, bleeding usually occurs because vascularised adhesions, consisting of aberrant vessels between the parietal and visceral pleura, are torn as the lung collapses following pneumothorax. The resultant massive haemorrhage and shock can be a surgical emergency.

SHP has been known since 1928, when it was diagnosed during an autopsy, and in the early 1970s, pleural aspiration first provided a successful non-operative treatment. ${ }^{3}$ Nowadays, thoracotomy $y^{4}$ is the surgical treatment of choice. Surgical interventions such as video assisted thoracic surgery (VATS) and thoracotomy have been valuable in decreasing the increase in mortality and morbidity resulting from the use of more conservative treatment regimens. ${ }^{56}$

In the early course of SHP, the clinical presentation is very similar to spontaneous pneumothorax without haemothorax (SP) and includes chest pain and dyspnoea, with pneumothorax in chest $x$ ray. However, if SHP remains unrecognised, the aforementioned life-threatening complications can ensue. The diagnosis of SHP has been hampered by lack of knowledge about the clinical presentation, risk factors, and its differentiation from SP. We undertook the present study to identify indicators and possible risk factors of SHP in patients with SP.

\section{MATERIALS AND METHODS}

Study population

We initially included all patients with primary SP attending the emergency department (ED) of Chi-Mei Foundation Medical Center, Tainan, Taiwan, between 1 January 1997 and
31 December 2002. The centre has approximately 120000 annual ED patient visits. Patients subsequently diagnosed as having haemopneumothorax caused by trauma or an underlying disease were excluded.

\section{Analyses}

In the remaining study population cases of SHP were identified by the aspiration of blood and air after tube thoracostomy in the absence of iatrogenic or other trauma to the chest. We collected demographic characteristics and data on clinical presentation (including initial symptoms and signs) and past medical history for all patients. Anthropometric measurements, including body weight and height, were recorded, and body mass index (BMI) was calculated as body weight divided by the square of body height $\left(\mathrm{kg} / \mathrm{m}^{2}\right)$. Blood samples were collected for laboratory tests upon arrival of the patient at the emergency department. The tests included haemoglobin levels and haematocrit, platelet count, prothrombin time, and the activated partial thromboplastin time (APTT). All patients also had a chest $x$ ray taken on arrival and before medical intervention. Board certified radiologists blind to the patients' diagnoses interpreted the images.

\section{Statistical analysis}

We used Fisher's exact test to evaluate the statistical significance of differences between categorical variables in the two study groups. For comparisons of continuous variables, Wilcoxon's rank sum test was used. All statistical

Abbreviations: APTT, activated partial thromboplastin time; ED, emergency department; PT, prothrombin time; SHP, spontaneous haemopneumothorax; SP, spontaneous pneumothorax without haemothorax; VATS, video assisted thoracic surgery 

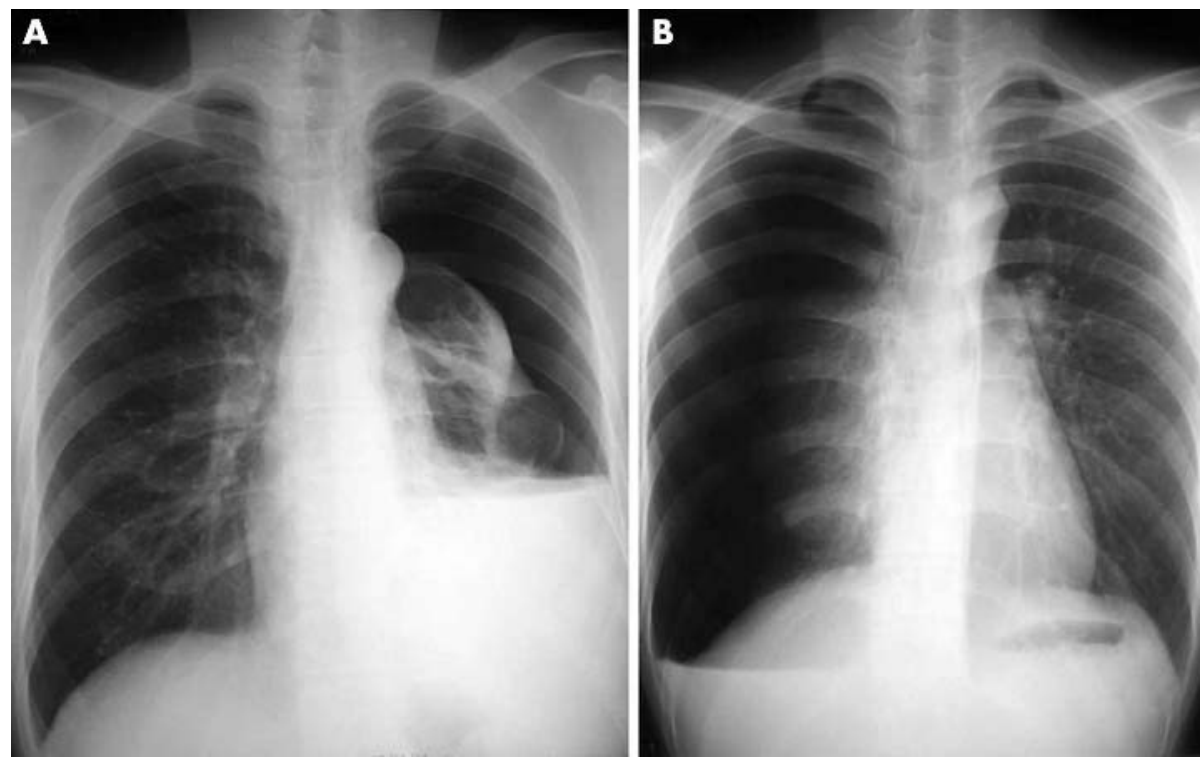

Figure 1 Chest $x$ ray findings in patients with spontaneous haemopneumothorax. (A) A pneumothorax with ipsilateral massive air-fluid level. (B) A pneumothorax with ipsilateral minimal air-fluid level. analyses were performed using the SAS software package (SAS, Cary, NC) at the two-sided significant level of 0.05.

\section{RESULTS}

Of the 211 patients with primary SP, eight $(3.79 \%)$ were diagnosed as having SHP. Only one patient had a past history of pneumothorax; the others had no past history of systemic or respiratory diseases. The chest $x$ rays revealed pneumothorax associated with an ipsilateral air-fluid level in all eight patients with SHP. Three patients presented with minimal air-fluid levels (defined as the blunting of the costal phrenic angle in the chest $x$ ray), and five with massive air-fluid levels (defined as greater than the blunting of costal phrenic angle) (fig 1). In contrast, pleural effusion was not observed in any of the patients with SP $(\mathrm{p}<0.01)$.

Blood (450-1770 ml) was drained immediately after tube thoracostomy (while still in the ED) in the five patients with massive air-fluid levels and hypovolaemic shock. In the three patients with minimal air-fluid levels, the blood was drained 75-375 minutes after tube thoracostomy. Surgical interventions were undertaken in seven patients after admission (VATS $(\mathrm{n}=4)$, mini-thoracotomy $(\mathrm{n}=1)$, and thoracotomy $(\mathrm{n}=2))$. The sources of bleeding identified were ruptured vascularised bullae $(n=5)$, aberrant vessel $(n=4)$, and lung parenchyma $(\mathrm{n}=1)$.

As shown in table 1 there was no significant difference between patients with SHP and SP with regard to sex (both were predominantly male), smoking, and presence of chest pain, but dyspnoea was more evident in patients with SHP than SP $(62.5 \% \vee 26.6 \%$, respectively; $\mathrm{p}=0.04)$. There was no

Table 1 Percentage comparison of categorical variables between patients with haemopneumothorax (SHP) and pneumothorax (HP)

\begin{tabular}{lllll}
\hline Variable & $\begin{array}{l}\text { SHP } \\
\mathbf{n}(\%)\end{array}$ & $\begin{array}{l}\text { SP } \\
\mathbf{n}(\%)\end{array}$ & $\begin{array}{l}\text { Odds ratio* } \\
\text { (95\% CI) }\end{array}$ & pt \\
\hline Male sex & $8(100.0)$ & $184(90.6)$ & $1.8(0.1$ to 32.3$)$ & $>0.95$ \\
Smoking & $6(75.0)$ & $102(50.5)$ & $2.9(0.6$ to 13.9$)$ & 0.28 \\
Dyspnoea & $5(62.5)$ & $54(26.6)$ & $4.6(1.1$ to 20.0$)$ & 0.04 \\
Chest pain & $8(100.0)$ & $179(88.2)$ & $2.3(0.1$ to 41.6$)$ & 0.60 \\
\hline
\end{tabular}

*Logit estimators were obtained using a correction of 0.5 in every cell when any of the cells had the value $0 ; \mathrm{Cl}$, confidence interval. tp value for Fisher's exact test.
Table 2 Range comparisons of categorical variables between patients with haemopneumothorax (SHP) and pneumothorax (HP)

\begin{tabular}{|c|c|c|c|}
\hline & SHP & SP & \\
\hline Variable & Mean (SD) & Mean (SD) & $\mathbf{p}^{*}$ \\
\hline Age & $24.0(7.1)$ & $28.8(13.6)$ & 0.46 \\
\hline Body weight $(\mathrm{kg})$ & $56.1(3.5)$ & $56.8(8.2)$ & $>0.95$ \\
\hline Height $(\mathrm{cm})$ & $177.4(5.4)$ & $170.3(6.0)$ & $<0.01$ \\
\hline Body mass index $\left(\mathrm{kg} / \mathrm{m}^{2}\right)$ & $17.9(1.7)$ & $19.6(2.5)$ & 0.06 \\
\hline Haemoglobin $(\mathrm{g} / \mathrm{dl})$ & $12.8(2.6)$ & $14.7(1.4)$ & 0.01 \\
\hline Haematocrit (\%) & $38.1(7.2)$ & $44.1(4.3)$ & $<0.01$ \\
\hline Prothrombin time† (seconds) & $11.8(0.8)$ & $12.4(1.6)$ & 0.16 \\
\hline International normalised ratio† & $1.0(0.1)$ & $1.0(0.1)$ & 0.31 \\
\hline APTT† (seconds) & $35.2(3.6)$ & $36.5(5.3)$ & 0.50 \\
\hline Platelets $\left(\times 10^{3} / \mathrm{mm}^{3}\right)$ & $275.6(164.7)$ & $252.8(63.6)$ & 0.39 \\
\hline Systolic blood pressure $(\mathrm{mm} \mathrm{Hg})$ & $136.1(18.9)$ & $129.5(18.4)$ & 0.38 \\
\hline Diastolic blood pressure $(\mathrm{mm} \mathrm{Hg})$ & $74.3(12.2)$ & $75.3(13.6)$ & 0.53 \\
\hline Heart rate (per minute) & $101.0(23.0)$ & $88.0(17.3)$ & 0.09 \\
\hline Respiratory rate (per minute) & $19.6(1.3)$ & 20.1 (1.7) & 0.33 \\
\hline
\end{tabular}

significant difference between the two groups as regards initial blood pressures, respiratory rate, and laboratory tests of prothrombin, international normalised ratio, APTT, and platelet levels (table 2). The difference in heart rate was marginally significant ( $101.0 \vee 88.0$ beats per minute in the SHP and SP groups, respectively, $\mathrm{p}=0.09$ ), and the SHP patients had significantly lower levels of haemoglobin and haematocrit compared with SP patients $(12.8 \vee 14.7 \mathrm{~g} / \mathrm{dl}$, $\mathrm{p}=0.01$; and $38.1 \% \vee 44.1 \%, \mathrm{p}<0.01$, respectively). The patients with SHP were generally younger, but the difference in age was not statistically significant $(p=0.46)$. Although the patients in the two groups had similar body weights, patients with SHP were taller than patients with SP ( $177.4 \mathrm{~cm} v 170.3 \mathrm{~cm}$, respectively, $\mathrm{p}<0.01$ ), and thus tended to have lower BMI $\left(17.9 \mathrm{~kg} / \mathrm{m}^{2} \vee 19.6 \mathrm{~kg} / \mathrm{m}^{2}\right.$, respectively, $\mathrm{p}=0.06)$.

\section{DISCUSSION}

Spontaneous haemopneumothorax is a rare disorder, which occurs in $1-12 \%$ of all spontaneous pneumothoraces, a range of $2-5 \%$ being the most common. ${ }^{12}$ Our finding of $3.8 \%$ is 
consistent with this. The condition can be life threatening due to the progressively massive blood loss into the pleural cavity leading to hypovolaemic shock. In addition to fluid resuscitation and stabilisation, surgical intervention is an appropriate treatment to minimise complications, specially in haemodynamically unstable patients. ${ }^{6-10}$ Five of our eight patients with SHP developed hypovolaemic shock in the ED and underwent emergency VATS or thoracotomy, and two underwent elective surgery because of persistent bleeding. The causes of bleeding were consistent with the known causes for SHP. ${ }^{8-12}$

The clinical picture of SHP is similar to that of SP. Because emergency physicians encounter the latter more often, there is a potential for overlooking SHP. We found that dyspnoea occurred more frequently in the patients with SHP, and this sign may be helpful in alerting medical staff to the possibility of SHP rather than SP.

Patients with SHP are at a higher risk of developing hypotension because of blood loss. None of our patients with SHP were hypotensive on arrival to the ED, and we did not find any statistically significant differences in the systolic or diastolic blood pressure between the SHP and SP groups. Therefore, signs of shock may be not prominent in patients with SHP in the early stage, and so the condition can be overlooked by clinicians until massive, life-threatening haemorrhage occurs. The higher, albeit marginally, heart rate in SHP patients may be another diagnostic indicator.

There was no evidence of bleeding tendency in the SHP group, and pertinent biochemical variables were all within normal limits and not significantly different from those of the SP group. Therefore, excessive and prolonged bleeding does not appear to be a significant risk factor in SHP. Nonetheless, on arrival at the ED, the patients with SHP had significantly lower levels of haemoglobin and haemocrit. Thus, the presence of anaemia or such abnormally low values represents another clinical indicator for SHP.

The radiological finding of pneumothorax associated with ipsilateral air-fluid level in the chest $x$ rays is the most important indicator of SHP. All of our eight SHP patients presented with pleural effusion. In contrast, none of the patients with SP had a similar finding. Therefore, clinicians should have a high index of suspicion of SHP in patients with radiographical findings of ipsilateral pleural effusions, especially in those with a massive air-fluid level.

In the present study blood was drained immediately after tube thoracostomy in all SHP patients with the chest $x$ ray finding of a massive air-fluid level. However, in those patients with minimal air-fluid levels, drainage was delayed up to 375 minutes. An explanation for the time difference may well be the large volume of blood lost into pleural cavity in association with massive pleural effusion. In patients with minimal air-fluid levels in the chest $x$ ray, there might have been no active bleeding soon after the onset of the condition or they might have just had mild oozing from a ruptured bulla or pleural adhesion following collapse of the lung.

Demographic features such as age, sex, and history of smoking offer no instructive diagnostic clues in the differentiation of SHP from SP. Furthermore, the reported increased male-to-female ratio in SHP than in $\mathrm{SP}^{13}{ }^{14}$ was not remarkable in the present study. However, our observations that patients with SHP were taller $(p<0.01)$ and tended to have lower BMI than patients with SP $(p=0.06)$ may offer reasonable clues to emergency physicians about the nature of the problem.

In conclusion, the clinical differences identified between SHP and SP in the present study, and, in particular, those evident on chest $x$ ray examination, offer a physician some means of diagnosing SHP earlier than is currently the case. Prompt and appropriate treatment may help avoid the lifethreatening complications that can ensue following a delay in diagnosis of SHP.

\section{Authors' affiliations}

C-C Hsu, Y-L Wu, H-J Lin, Department of Emergency Medicine, Chi-Mei Foundation Medical Center, Tainan, Taiwan

M-P Lin, Department of Radiology, Chi-Mei Foundation Medical Center, Tainan, Taiwan

H-R Guo, Department of Environmental and Occupational Health, Medical College, National Cheng Kung University, Tainan, Taiwan

Competing interests: none declared

\section{REFERENCES}

1 Abyholm FE, Storen G. Spontaneous hemopneumothorax. Thorax 1973;28:376-8.

2 O'Neill S. Spontaneous pneumothorax: aetiology, management and complications. Ir Med J 1987;80:306-11.

3 Andreassian B, Peyredieu G, Nussaume O, et al. Hemopneumothorax et hemothorax dits spontanes: a propos de 21 cases. Ann Chir Thorac Cardiovasc 1971; 10:367-73.

4 Elrod PD, Murphy JD. Spontaneous hemopneumothorax treated by decortication. J Thorac Surg 1948;17:401.

5 Deaton WR, Johnston FR. Spontaneous hemopneumothorax. J Thorac Cardiovasc Surg 1962;43:413-15.

6 Tatebe S, Kanazawa H, Yamazaki Y, et al. Spontaneous hemopneumothorax. Ann Thorac Surg 1996;62:1011-15.

7 Tatebe S, Yoshiya K, Yamaguchi A. Video-assisted thoracoscopic surgery for spontaneous hemopneumothorax. Surg Laparosc Endosc 1997;7:113-15.

8 Eastridge CE. Spontaneous hemopneumothorax requiring thoracotomy. South Med J 1985;78:1392-3.

9 Myers RT, Johnston FR, Bradshaw HH. Spontaneous hemopneumothorax: report of a case treated by thoracotomy. Ann Surg 1951;133:413-16.

10 Williams MH, Carmen JC, Seymour DM. Emergency thoracotomy for massive spontaneous hemopneumothorax. N Engl J Med 1954;251:888-91.

11 Fry W, Rogers WL, Crenshaw GL, et al. The surgical treatment of spontaneous idiopathic hemopneumothorax; a review of the published experience with a report of thirteen additional cases. Am Rev Tuberc 1955;71:30-48.

12 Ross CA. Spontaneous hemopneumothorax. J Thorac Surg 1952;23:582-92.

13 Light RW. Management of spontaneous pneumothorax. Am Rev Respir Dis 1993; 148:245-8.

14 Melton L, Hepper NGG, Offord KP. Incidence of spontaneous pneumothorax in Olmsted County, Minnesota: 1950-1974. Am Rev Respir Dis 1974; 120:1379-82. 